\title{
The complex relationship between CD4 count, HIV viral load, trimethoprim-sulfamethoxazole prophylaxis, and skin-and-soft- tissue infection risk in patients with HIV: insights from a causal diagram and simulation study
}

\author{
V. HEMMIGE ${ }^{1 *}$, D. S. LAUDERDALE ${ }^{2}$ AND M. Z. DAVID ${ }^{2,3,4}$ \\ ${ }^{1}$ Division of Infectious Diseases, Department of Medicine, Baylor College of Medicine, Houston, TX, USA \\ ${ }^{2}$ Department of Public Health Sciences, University of Chicago, Chicago, IL, USA \\ ${ }^{3}$ Section of Infectious Diseases and Global Health, Department of Medicine, University of Chicago, Chicago, IL, USA \\ ${ }^{4}$ Department of Pediatrics, University of Chicago, Chicago, IL, USA
}

Received 14 December 2015; Final revision 31 March 2016; Accepted 1 April 2016;

first published online 4 May 2016

\section{SUMMARY}

Skin and soft tissue infection (SSTIs) due to Staphylococcus aureus, particularly communityassociated methicillin-resistant Staphylococcus aureus (CA-MRSA), are common in human immunodeficiency virus (HIV)-infected populations in the United States. Studies have differed as to the importance of epidemiological and immunological factors in this relationship, and have employed conflicting strategies for variable selection in multivariate analyses. Developments in causal inference methods in epidemiology have emerged in the last decade to clarify relationships between variables and identify appropriate variables to include in and exclude from multivariate analysis. In this paper, we develop a causal diagram to clarify the pathways linking CA-MRSA and HIV. We focus on the role played by trimethoprim-sulfamethoxazole (TMP-SMX) prophylaxis, prescribed to many severely immunocompromised HIV patients and potentially protective against SSTIs, which both mediates and moderates the relationship between immunological parameters and SSTI risk. We demonstrate, using simulated data, that statistical models may yield biased results if they do not account for how HIV viral load may also be a marker of adherence to TMP-SMX prophylaxis. We conclude with a proposed causal model that includes both the epidemiological as well as immunological factors that may explain the increased risk of initial and recurrent SSTI risk in HIV-infected populations.

Key words: AIDS, epidemiology, methicillin - S. aureus resistant to (MRSA), skin infections.

\section{INTRODUCTION}

Skin and soft tissue infections (SSTIs) due to Staphylococcus aureus have plagued humans since antiquity [1]. The development of penicillin [2] and then, subsequently, anti-staphylococcal penicillins such as methicillin [3] offered the option of pharmacotherapy

\footnotetext{
* Author for correspondence: Dr V. Hemmige, Baylor College of Medicine, Baylor Clinic, 6620 Main, Suite 1375, Houston, TX 77030, USA.

(Email: vagish.hemmige@bcm.edu)
}

against these pathogens. In 1961, however, the first strains of methicillin-resistant $S$. aureus (MRSA) were described [4]. These strains quickly became endemic in hospitals and intensive care units throughout the 1970s and 1980s, causing infections in debilitated and chronically ill individuals. They also caused occasional outbreaks in populations having close contact with the healthcare system such as intravenous drug users. However, they were not seen to cause outbreaks in healthy outpatients [5].

Since the first discovery of US strains of communityassociated (CA)-MRSA 15 years ago [6], a marked 
increase in the incidence of SSTIs has been noted in outpatient populations [7]. These strains differ from previous prevalent strains of $S$. aureus not only in their drug resistance pattern, but also in their virulence [5]. These pathogens have been noted to cause recurrent infections even in otherwise seemingly healthy individuals, and the burden of CA-MRSA infections has fallen particularly on marginalized populations [8].

Even before the advent of CA-MRSA, populations infected with human immunodeficiency virus (HIV) were particularly susceptible to SSTIs [9]. However, in the last 10 years, a number of reports have described the disproportionate impact of CA-MRSA on people with HIV, even those with seemingly intact immune systems as measured by CD4 lymphocyte counts $[10,11]$. A high rate of recurrence of these infections has been observed in this population $[12,13]$. Both immunological factors and epidemiological factors may determine risk of SSTIs in HIVinfected populations [14, 15]. Furthermore, studies on recurrent SSTIs in HIV-infected populations have suggested that the risk factors for recurrent SSTIs may differ from those for initial SSTI $[13,16]$.

Previous work has identified conflicting risk factors for CA-MRSA SSTIs in the HIV-infected population [10], and the epidemiology remains far from understood. Studies have shown conflicting results as to whether being immunocompromised, as measured by CD4 count, is a risk factor for CA-MRSA SSTI [17-20]. A complicating factor in assessing the relationship between CD4 count and SSTI risk is that trimethoprim-sulfamethoxazole (TMP-SMX) is typically prescribed to non-allergic HIV-infected patients with CD4 lymphocyte count $<200$ cells/ml to prevent Pneumocystis pneumonia as per standardized guidelines [21]. TMP-SMX also demonstrates microbiological activity against most strains of $S$. aureus, including CA-MRSA [22] and several other bacterial species that are common causes of SSTIs, and studies have yielded conflicting results as to the role that TMP-SMX prophylaxis plays in altering SSTI risk [17-19]. Studies have also differed as to the impact of a low HIV viral load, achieved through the use of antiretroviral therapy (ART), on mitigating SSTI risk in this population [23].

Causal diagrams, also known as directed acyclic graphs, frequently aid in clarifying complicated epidemiological relationships and in designing multivariable analyses [24]. In a causal diagram, the outcome variable, exposures of interest, and potential confounders are included as nodes. Then, a one-way arrow between two variables is added if and only if a truly causal relationship is believed to exist between the two variables. Statistical associations between variables which are not directly causal are not depicted in causal diagrams [24]. Sets of rigorous mathematical rules exist to determine, based on the structure of a causal diagram, which variables should be included in an adjusted analysis, and which should be excluded [25]. This task has been greatly facilitated by recently developed software [26].

Previous studies have treated TMP-SMX prophylaxis as a traditional confounder [19]. The relationship between CD4 count, HIV viral load, TMP-SMX exposure, and SSTI risk is more complicated than previous authors have appreciated; however, TMP-SMX prophylaxis is not a confounder by traditional epidemiological definitions. To demonstrate this, we developed a causal diagram for the association between TMP-SMX use, immunological parameters, and SSTI risk in an HIV-infected population (Fig. 1). For TMP-SMX use to serve as a confounder of the relationship between CD4 count and SSTI risk, it would need to be associated with the explanatory variable (CD4) as well as the outcome (SSTI), and not lie on a causal pathway between CD4 count and SSTI risk [27]. However, a low CD4 count directly causes use of TMP-SMX, i.e. it triggers the prescription of TMP-SMX by a healthcare provider. Therefore, the actual relationship is that of competing causal pathways (Fig. 1a). In one causal pathway, a low CD4 count increases SSTI risk by causing immunological impairment. In an alternate pathway, a low CD4 count decreases SSTI risk by causing the use of TMP-SMX prophylaxis. Both are technically causal effects of CD4 count. The total effect of CD4 count is the sum of these two indirect effects, one mediated by immunological mechanisms, and the other by TMP-SMX prophylaxis. However, most previous authors have presumably only intended to consider the former as the 'causal' effect of CD4 count upon SSTI risk in their analyses. However, adjusting for TMP-SMX use, at first glance, may still seem to be appropriate, as this may isolate the immunological effect of a low CD4 count [28], the presumed goal of previous authors.

However, when we include other variables associated with HIV status such as viral load and its predictors in the analysis the relationship is further complicated (Fig. 1b). HIV viral load is typically considered an independent marker of immune function, 
(a)
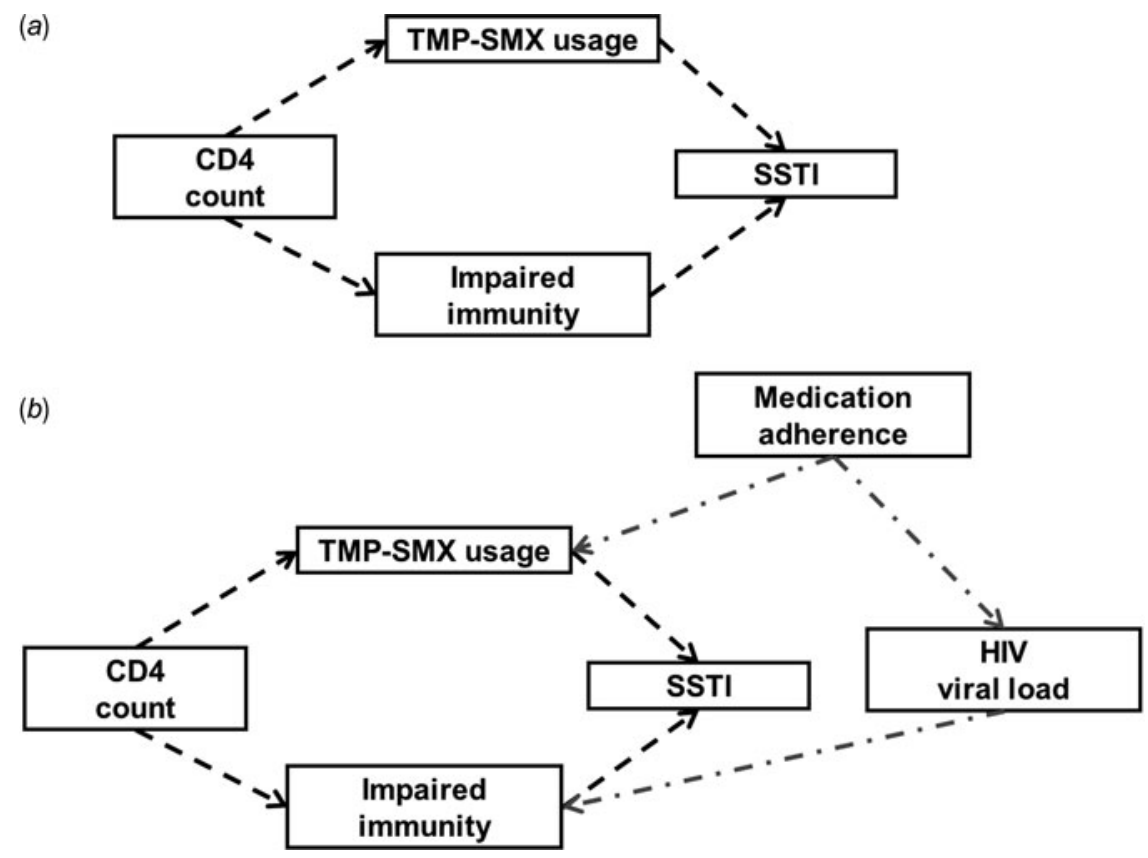

Fig. 1. (a) Causal diagram for the relationship between CD4 count, HIV viral load, trimethoprim-sulfamethoxazole (TMP-SMX) prophylaxis, and skin and soft tissue infection (SSTI) risk. Note the location of TMP-SMX usage on one causal pathway between CD4 count and SSTI risk, so that TMP-SMX use is not a confounder. (b) TMP-SMX use itself is affected by medication adherence, which has a causal impact on HIV viral load as well.

since patients with low CD4 counts and low viral loads typically experience lower rates of opportunistic infection than patients with equivalent CD4 counts but higher viral loads [29]. However, HIV viral load is strongly associated with medication adherence to ART. If adherence to ART is associated with adherence to other medications, a plausible although to our knowledge not validated assumption, then HIV viral load may serve as a marker of adherence to TMP-SMX prophylaxis. Accordingly, HIV viral load may serve as a modifier of the effect of TMPSMX prophylaxis on SSTI risk, since a high viral load would mark non-adherent patients who would be expected to derive little benefit from being prescribed TMP-SMX prophylaxis as they will not actually take the medication. In other words, there may be an interaction between HIV viral load and TMP-SMX prescription in their association with risk of SSTI. Some previous studies that have found low HIV viral load significantly predicted lower SSTI risk did not adjust for TMP-SMX use, and part of the perceived effect of viral load may have been as a marker of adherence to TMP-SMX therapy.

Having clarified the complex relationship between CD4 count, viral load, TMP-SMX prophylaxis, and SSTI risk, we developed a simulation in this paper to demonstrate the potential bias in analyses which fail to account for these complicated relationships.

\section{METHODS}

We developed a basic simulation of SSTI occurrence in HIV-infected populations using Stata v. 12 (StataCorp., USA). The code is available in the Supplementary material. We assumed a cohort of 2000 subjects, $30 \%$ of whom have a CD4 count of $<200$ cells $/ \mathrm{ml}$. We assumed that $80 \%$ of individuals with CD4 count of $<200$ cells $/ \mathrm{ml}$ were prescribed TMP-SMX prophylaxis, and that the rest had a contraindication to receiving the medication (e.g. due to allergies). No one with CD4 $\geqslant 200$ cells $/ \mathrm{ml}$ was assumed to be prescribed TMP-SMX prophylaxis. Eighty per cent of those with CD4 count $>200$ cells $/ \mathrm{ml}$, but just $50 \%$ of those with CD4 count $<200$ cells $/ \mathrm{ml}$, were assumed to have HIV viral load $<1000$ copies/ml. We assumed that virologically suppressed patients were adherent to TMP-SMX prophylaxis, but that only $25 \%$ of non-suppressed patients who were prescribed TMP-SMX were taking it. We assumed, for the purposes of this simulation, that subjects with CD4 count $<200$ cells $/ \mathrm{ml}$ experienced a risk of SSTI which was triple those with higher CD4 
counts, that subjects with HIV viral load $<1000$ copies $/ \mathrm{ml}$ experienced a risk of SSTI which was half that seen in subjects with higher viral loads, and that TMP-SMX use would decrease the daily risk of SSTI by $30 \%$. These numbers were chosen arbitrarily for the purposes of illustration. We calculated the daily probability of SSTI based on the CD4 count, HIV viral load, and actual TMP-SMX use status, the daily risk of SSTI, and the ratios in Table 1. No variables were assumed to be time-varying, and no censoring was present. The simulation was run 100 times. We did not simulate other factors associated with SSTI risk as the goal of this simulation was to demonstrate the effect of various strategies (some correct, some incorrect) of accounting for the complex relationship between CD4 count, HIV viral load, TMP-SMX prophylaxis, and SSTI risk on regression analysis results.

For each simulated dataset, we proceeded with a series of Cox regression models, using time to first SSTI as the outcome and CD4 count, HIV viral load, and either prescription of TMP-SMX (the variable available in a clinical study) or actual use of TMP-SMX (additional data typically not available in a real-world study) as covariates [30]. Models 111 included varying combinations of the four covariates, and models 12 and 13 included interaction terms between viral load and TMP-SMX prophylaxis as well. The exponentiated means of the coefficient of the covariates from the 100 simulations under each model were calculated, and forest plots of the confidence intervals of the hazard ratios of the covariates from each simulation and each model are included in the Supplementary material. For each of the models, we assessed the difference between the actual value of the parameters we sought to estimate and the estimate yielded under the model.

In our Discussion below, we assumed, in regards to the CD4 count variable, if included in the model, that the goal of analysis was to estimate the immunological effect of CD4 count on SSTI risk, not the total effect. We assumed that, if included in the model, the goal of analysis was to obtain unbiased estimates of the total effects of HIV viral load and actual TMP-SMX use on SSTI risk.

\section{RESULTS}

In the 100 simulations, a mean of $454(23 \%)$ of 2000 subjects experienced an SSTI during 5 years of followup, comparable to observed results in clinical cohorts
Table 1. Parameters in the simulation

\begin{tabular}{ll}
\hline \hline Parameter in simulation & Value \\
\hline Size of cohort & 2000 subjects \\
Duration of follow-up & 5 years \\
Proportion of subjects with CD4 & $30 \%$ \\
$<200$ cells/ml & \\
Proportion of subjects virologically & \\
suppressed & $50 \%$ \\
CD4 <200 cells/ml & $80 \%$ \\
CD4 $\geqslant 200$ cells/ml & $80 \%$ \\
Probability of TMP-SMX prescription if & \\
CD4 <200 cells/ml & \\
Probability of TMP-SMX adherence & $100 \%$ \\
HIV viral load <1000 copies/ml & $25 \%$ \\
HIV viral load $\geqslant 1000$ copies/ml & $0 \cdot 000125 /$ day \\
Baseline risk of SSTI & \\
Hazard ratio of SSTI* & 3 \\
CD4 <200 cells/ml (immunological effect) & $0 \cdot 5$ \\
HIV viral load $<1000$ copies/ml & $0 \cdot 7$ \\
TMP-SMX use & \\
\hline \hline
\end{tabular}

TMP-SMX, Trimethoprim-sulfamethoxazole; SSTI, skin and soft tissue infection.

* This is the independent multiplicative increase in risk associated with each factor. That is to say, those with CD4 count $<200 \mathrm{cell} / \mathrm{ml}$ experience a risk of SSTI which is triple that observed in subjects with a higher CD4 count, subjects with HIV viral load $<1000$ copies $/ \mathrm{ml}$ experience a risk of SSTI which is half that seen in subjects with higher viral loads, and TMP-SMX users experience a risk of SSTI which is $0 \cdot 7$-fold that seen in subjects not using TMP-SMX.

[18]. The summary results of the regression analyses on the simulated data are shown in Table 2. In model 1 , in which we had perfect knowledge as to whether or not the patients were adherent to TMP-SMX prophylaxis, we attained unbiased estimates of the effect of CD4 count, HIV viral load, and TMP-SMX use when we included all three in the statistical model. Model 2, with CD4 count alone as a predictor, achieved a reasonably close estimate to the true effect of CD4 count on SSTI risk. Model 3, with HIV viral load alone, was biased towards demonstrating a stronger effect of virological suppression on SSTI than is in fact the case. This is because, in this model, the coefficient for HIV viral load partially captured the effect of adherence to TMP-SMX prophylaxis. Models 4 and 5, with TMP-SMX prescription and use alone, respectively, were qualitatively biased and erroneously suggested that TMP-SMX is associated with an increased risk of SSTI due to confounding by CD4 count and HIV viral load. Model 6 adjusts for CD4 count and viral load, but makes no attempt to adjust for the effect 
Table 2. Geometric mean values of hazard ratios associated with predictors of initial SSTI over 100 simulations in univariate and multivariate Cox regression models without interaction terms

\begin{tabular}{|c|c|c|c|c|c|c|c|c|c|c|c|}
\hline Factor & $\begin{array}{l}\text { Model } \\
1\end{array}$ & $\begin{array}{l}\text { Model } \\
2\end{array}$ & $\begin{array}{l}\text { Model } \\
3\end{array}$ & $\begin{array}{l}\text { Model } \\
4\end{array}$ & $\begin{array}{l}\text { Model } \\
5\end{array}$ & $\begin{array}{l}\text { Model } \\
6\end{array}$ & $\begin{array}{l}\text { Model } \\
7\end{array}$ & $\begin{array}{l}\text { Model } \\
8\end{array}$ & $\begin{array}{l}\text { Model } \\
9\end{array}$ & $\begin{array}{l}\text { Model } \\
10\end{array}$ & $\begin{array}{l}\text { Model } \\
11\end{array}$ \\
\hline $\begin{array}{l}\text { CD4 count } \\
<200 \text { cells } / \mathrm{ml}\end{array}$ & $3 \cdot 02$ & $3 \cdot 15$ & & & & $2 \cdot 51$ & $2 \cdot 92$ & $4 \cdot 44$ & 3.67 & & \\
\hline $\begin{array}{l}\text { HIV viral load } \\
<1000 \text { copies } / \mathrm{ml}\end{array}$ & $0 \cdot 51$ & & $0 \cdot 35$ & & & $0 \cdot 45$ & $0 \cdot 45$ & & & $0 \cdot 34$ & $0 \cdot 41$ \\
\hline $\begin{array}{l}\text { TMP-SMX } \\
\text { prescription }\end{array}$ & & & & & $2 \cdot 57$ & & $0 \cdot 84$ & & $0 \cdot 84$ & & $2 \cdot 03$ \\
\hline TMP-SMX use & 0.69 & & & $1 \cdot 39$ & & & & $0 \cdot 47$ & & $1 \cdot 57$ & \\
\hline
\end{tabular}

TMP-SMX, Trimethoprim-sulfamethoxazole; SSTI, skin and soft tissue infection.

Results of various Cox regression modelling strategies applied to our simulated data set. Model 1, in which we know and adjust for whether TMP-SMX is actually used as well as CD4 count and HIV viral load, is unbiased and thus us the standard to which subsequent models can be compared for evidence of bias. Model 2, in which only CD4 count is included in the model, yields a slightly upward biased estimate for the effect of CD4 count. Model 3, in which we only account for HIV viral load, overestimates the effect of virological suppression on SSTI risk. Models 4 and 5, in which we only account for TMP-SMX prescription and actual receipt without accounting for CD4 and HIV viral load, are highly biased and suggest TMP-SMX increases the risk of SSTI. Model 6, in which we account for CD4 count and HIV viral load but not TMP-SMX use, underestimates the immunological effect of low CD4 count on SSTI risk. Model 7, where we do not have knowledge of TMP-SMX adherence, underestimates the protective effect of TMP-SMX and yields slightly biased estimates for the effect of CD4 and HIV viral load as well. Models 8 and 9, where we only account for CD4 count and TMP-SMX use, overestimate the effect of CD4 count. Models 10 and 11, where we account for HIV viral load and TMP-SMX prophylaxis but not CD4 count, erroneously suggest that TMP-SMX use increases SSTI risk.

of TMP-SMX; we observe that the estimated effect of CD4 count is biased towards the null in this scenario.

In model 7, where we used TMP-SMX prescription alone and did not know if patients were adherent or not to therapy, while adjusting for CD4 count and HIV viral load, we obtained downwardly biased estimates of the effect of low CD4 and high viral load on SSTI risk. In this model, the estimate of the effect of TMP-SMX on SSTI risk was biased towards suggesting that TMP-SMX prophylaxis is less effective at preventing SSTIs than is actually the case.

Models 8 and 9 showed that, when we included CD4 count and TMP-SMX use in a model without HIV viral load, we overestimated the effect of CD4 count on SSTI risk. When actual TMP-SMX use was the covariate, we overestimated the protective effect of TMP-SMX use; when we used TMP-SMX prescription as the covariate, we underestimated the protective effect of TMP-SMX use. In models 10 and 11 , in which we included HIV viral load and TMP-SMX use but not CD4 count as covariates, we found that we overestimated the protective effect of virological suppression and erroneously concluded that TMP-SMX is associated with increased SSTI risk.

In model 12 (Table 3), in which we included a term to account for the interaction between HIV viral load and the effectiveness of TMP-SMX prescription, we found that we obtained more accurate estimates of the effect of CD4, viral load, and TMP-SMX use on SSTI risk compared to model 7, which did not include the interaction term. Interestingly, despite the interaction term between HIV viral load and TMP-SMX prescription in model 12 being necessary for an unbiased estimate of the true effect of TMP-SMX exposure on SSTI risk, the interaction term itself was only statistically significant in 31/100 simulations. Model 13 included an interaction term between HIV viral load and the effectiveness of TMP-SMX use itself and obtained unbiased estimates of the coefficients. However, in the case when it is actually known whether or not subjects were adherent to TMPSMX, the interaction term was unnecessary, as model 1, which included CD4 count, HIV viral load, and TMP-SMX use, but no interaction term, was already an unbiased model. However, the inclusion of the interaction term did not introduce bias into the model.

\section{DISCUSSION}

In this paper, we have developed a causal diagram to depict the complex relationship between CD4 count, HIV viral load, TMP-SMX prophylaxis, and SSTI 
risk in HIV patients. We have used a basic simulation to show that failure to account for the interaction between HIV viral load and TMP-SMX prescription may lead to biased results, as subjects with high HIV viral load may be less likely to be adherent to TMP-SMX and so the effect of TMP-SMX prescription on SSTI risk may be attenuated in this group. As noted, in model 12, even though the interaction term itself only reached conventional levels of statistical significance $(P<0.05)$ in a minority of simulations, inclusion of the interaction term was necessary to avoid biased results. This is consistent with the advice of authors who have warned against using statistical significance alone as a marker of whether an interaction term should be included in nonlinear models, as most real-world studies featuring binary outcomes are underpowered to detect interaction [31]. It is likely that the power of any feasible real-world cohort study to detect an interaction between HIV viral load and TMP-SMX prescription on SSTI risk will be significantly less than the already low power seen in this simulation. However, our simulation demonstrates the concept already suggested by the causal diagram in Figure $1 b$, that the interaction should be accounted for based on theoretical concerns alone.

In the above discussion, we have not addressed the question of whether a subject has a high viral load because of non-adherence to ART or because ART has not yet been prescribed to them. This is primarily an historical issue, as current practice in developed countries would recommend ART for all HIV-diagnosed subjects [32] and even previous practice guidelines would have required ART initiation for any HIVdiagnosed subject for whom Pneumocystis prophylaxis was indicated. No biological mechanism has been identified by which ART would exert a direct effect on SSTI risk independent of its indirect effect mediated via viral load. Accordingly, we do not explicitly model whether high HIV viral load is due to lack of ART prescription or due to non-adherence in our analysis.

Although the assumption of virologically nonsuppressed patients demonstrating lower adherence to TMP-SMX prophylaxis than virologically suppressed patients seems highly plausible, we are not aware of any study in the HAART era in the United States which explicitly stratifies Pneumocystis prophylaxis adherence by HIV viral load. One study from the pre-HAART era in an urban, Medicaid-insured population which was $\sim 60 \%$ adherent to antiretrovirals found that $80 \%$ of patients receiving Pneumocystis prophylaxis were using TMP-SMX as opposed to alternative agents for Pneumocystis prophylaxis, such as dapsone and atovaquone, which do not have antistaphylococcal activity. Adherence to Pneumocystis prophylaxis overall, however, was $<50 \%$ [33].

Of note, the situation depicted in Figure $1 b$, with confounding of an intermediate in a causal pathway, is one in which other authors, based on the structure of the causal diagram, have noted that standard regression will lead to biased estimates [34]. In this setting, an understanding of the causal diagram immediately identifies the potential for bias in the analysis if care is not taken to account for this interaction. We refer readers interested in further details on causal diagrams to a recent review of this topic that focuses on applications to clinical research [35].

We did not account for longitudinal changes over time in the above, simplified model. This relationship would be further complicated, as present HIV viral load and present CD4 count would both impact future CD4 count, which would in turn impact future TMP-SMX usage. Standard regression methods are known to be prone to bias when dealing with complex time-varying predictors, as the same covariate can serve as both a confounder and mediator simultaneously [36]. Time-varying confounding is an area of active research in statistics, with marginal structural models one method potentially offering advantages over standard longitudinal analytic methods in minimizing bias [37]. Further discussion of this topic, however, is beyond the scope of this paper. Similarly, we employed a regression model using time to first SSTI as an outcome to avoid the complexities of recurrent events analysis, a topic well-reviewed in previous papers [38] which is also outside the scope of the current paper.

We conclude by attempting to develop a causal analysis model, applicable to all populations at risk for MRSA infections (Fig. 2a). Initial MRSA exposure leads to initial colonization. Initial MRSA colonization leads to an increased risk of initial MRSA infection as well as subsequent MRSA colonization, which leads to subsequent (recurrent) MRSA infection. Subsequent (recurrent) MRSA exposure also contributes to the risk of subsequent MRSA colonization. Accordingly, factors that contribute to elevated SSTI risk in the HIV-infected population must do so by affecting at least one portion of the above pathway.

We propose a model for MRSA SSTI risk in the HIV-infected population in Figure 2(b, c). Epidemiological factors may lead to increased MRSA exposure. 
(a)
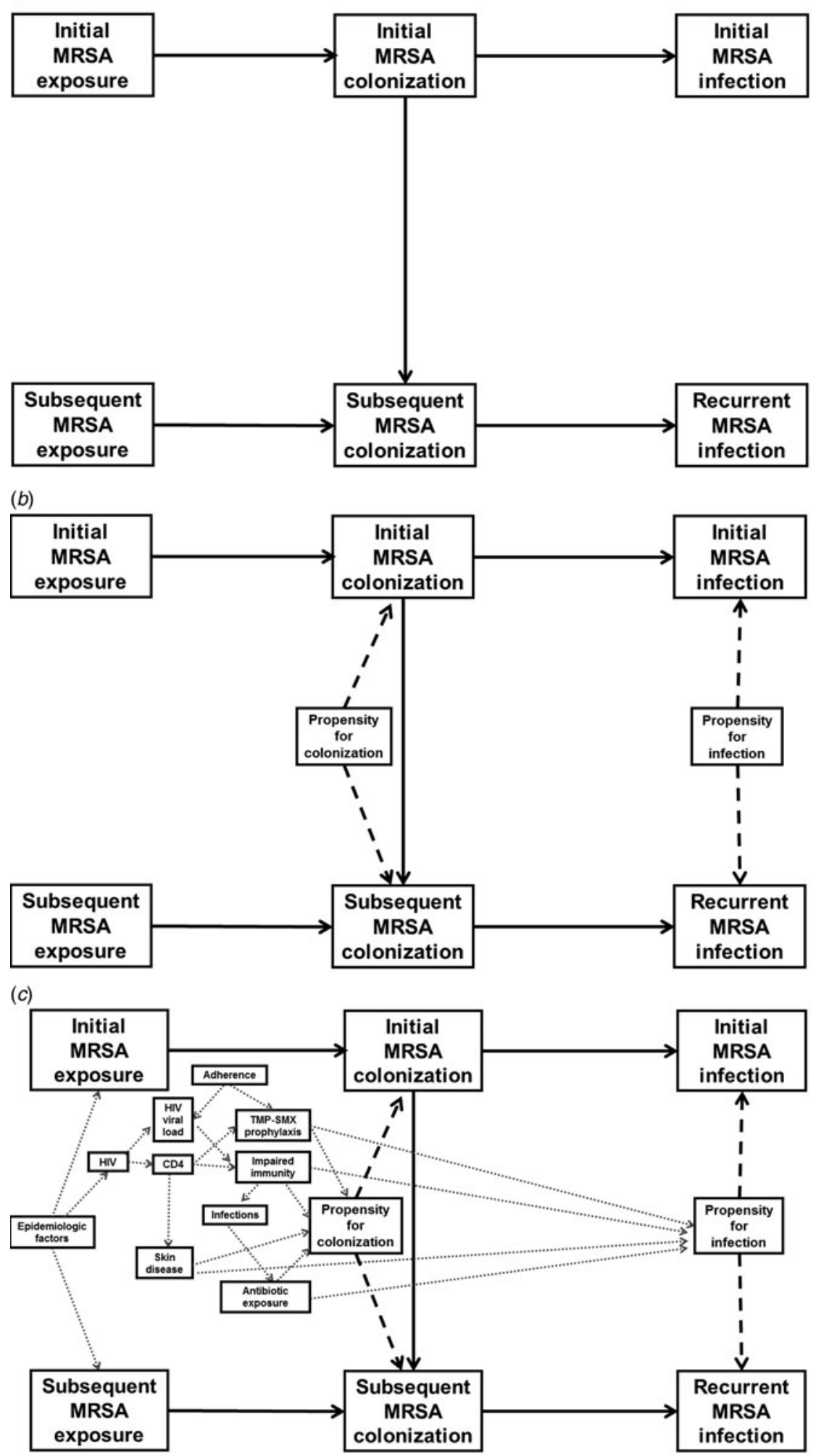

Fig. 2. (a) Skeletal causal diagram of key events in initial and subsequent methicillin-resistant Staphylococcus aureus (MRSA) infections, applicable to all populations. (b) Propensity for colonization and infection added to panel $(a)$. $(c)$ The key immunological and medical parameters specific to HIV populations have been added to the skeletal causal diagram in panel $(b) .(d)$ Key epidemiological links between HIV and MRSA. Some factors (e.g. sexual risk factors) are likely directly causal for both HIV and for MRSA exposure. Other epidemiological factors (e.g. crowded living) are likely to be associated with HIV due to an undetermined underlying cause. 


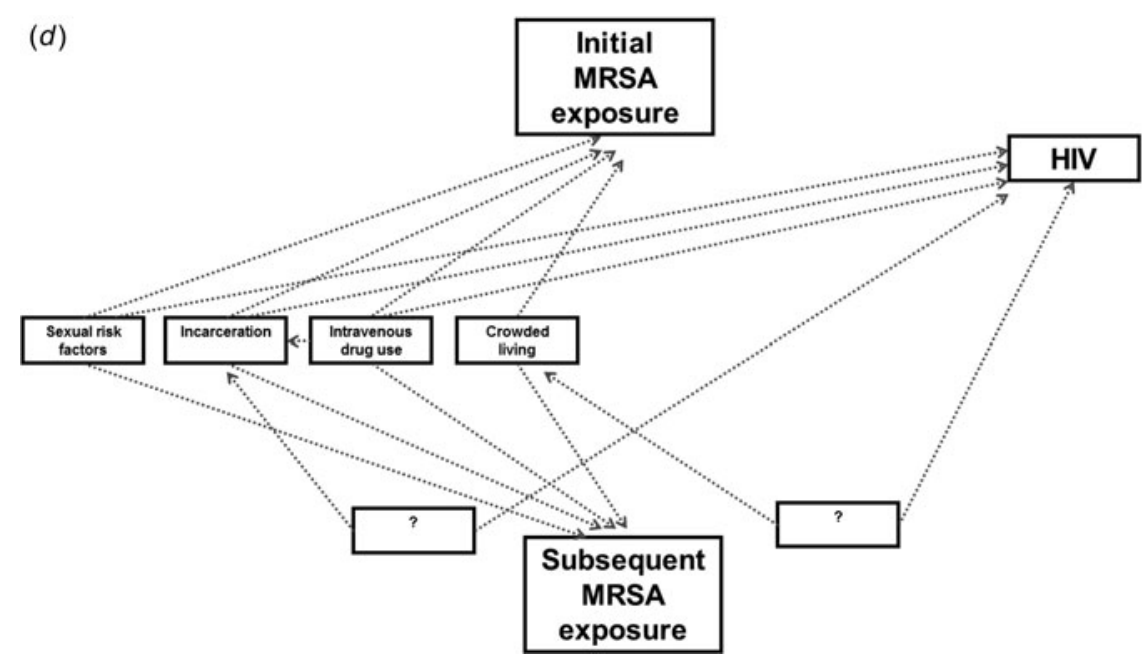

Fig. 2. (cont.). For legend see previous page.

Table 3. Geometric mean values of hazard ratios associated with predictors of initial SSTI over 100 simulations in univariate and multivariate Cox regression models with an interaction term

\begin{tabular}{lll}
\hline \hline Factor & Model 12 & Model 13 \\
\hline CD4 <200 cells/ml & $3 \cdot 02$ & $3 \cdot 02$ \\
HIV viral load <1000 copies/ml & $0 \cdot 51$ & $0 \cdot 51$ \\
TMP-SMX prescription & & \\
If HIV viral load $\geqslant 1000$ copies $/ \mathrm{ml}$ & $0 \cdot 93$ & \\
If HIV viral load <1000 copies $/ \mathrm{ml}$ & $0 \cdot 69$ & \\
TMP-SMX use & & \\
If HIV viral load $\geqslant 1000$ copies $/ \mathrm{ml}$ & & $0 \cdot 70$ \\
If HIV viral load <1000 copies/ml & 0.69 \\
\hline \hline
\end{tabular}

TMP-SMX, Trimethoprim-sulfamethoxazole; SSTI, skin and soft tissue infection.

Results of various Cox regression modelling strategies utilizing interaction terms applied to our simulated dataset. We see that model 12, which introduces an interaction between HIV viral load and TMP-SMX prescription to model 7, corrects much of the bias seen in that model. Model 13, which introduces an interaction between HIV viral load and TMP-SMX prescription to model 1, preserves the unbiased nature of that model.

Both epidemiological and immunological factors may contribute to an increased risk of an exposure to MRSA leading to colonization. Subsequently, either epidemiological or immunological factors may contribute to an increased likelihood of infection in MRSA-colonized individuals who are HIV-infected. The same or other factors may contribute to an increased likelihood of persistent colonization or persistent re-exposure leading to re-colonization in the future, leading to subsequent infection. Figure $2 c$ includes some of the details of how we propose that HIV-specific immunological issues may potentially interact to promote prolonged duration of MRSA colonization and progression from colonization to active infection. Specifically, skin disease, which is more prevalent at low CD4 counts, may be an intermediary of one causal pathway between CD4 count and SSTI risk, as noted by previous authors [39].

We further elucidate potential epidemiological factors that potentially may lead to increased MRSA exposure risk in the HIV-infected population. These are all listed in Figure $2 d$, where we have added sexual risk factors, incarceration, intravenous drug use, and crowded living. All of the above have been identified as potential epidemiological risk factors for MRSA exposure or infection in patients with HIV infection $[11,15]$. Some risk factors are likely directly causal. For example, high-risk sexual practices likely lead directly to MRSA exposure as well as exposure to HIV. Other risk factors such as crowded housing are likely to be directly causal for MRSA exposure, but only related to HIV via an undetermined factor that leads to both HIV infection and living in crowded housing. Incarceration, a well-known risk factor for MRSA infection, potentially is linked to HIV directly (through HIV acquisition in prison), as well as indirectly, with drug use leading to incarceration as well as HIV acquisition being one potential indirect pathway.

\section{CONCLUSION}

A complicated interplay between epidemiological factors, immunological factors, and antibiotic exposure including TMP-SMX prophylaxis may explain the 
increased risk of MRSA colonization and infection in HIV-infected populations. Causal diagrams reveal that statistical models which only account for CD4 count, HIV viral load, and TMP-SMX prescription as confounders and do not account for the complicated interactions among these variables with each other and with adherence may yield biased results. We were able to demonstrate this bias with a simulation study. We proposed a causal diagram that clarifies underlying relationships and aids in identifying appropriate variables to include in future statistical analyses of SSTI risk in HIV-infected populations. As new potential determinants of SSTI risk in this population are identified, their relationship to previously identified risk factors will need to be carefully considered in order to yield unbiased estimates though multivariable models. Our example of the use of causal diagrams, combined with a consideration of the relationship of specific putative risk factors to one another, may be applicable to studies of other infectious diseases in other populations.

\section{SUPPLEMENTARY MATERIAL}

For supplementary material accompanying this paper visit http://dx.doi.org/10.1017/S0950268816000789.

\section{ACKNOWLEDGEMENTS}

Michael David acknowledges funding from NIH NIAID grant K23 AI095361.

\section{DECLARATION OF INTEREST}

None.

\section{REFERENCES}

1. Cook L, Cook KF. Deadly Diseases and Epidemics: Staphyloccus aureus Infection. Philadelphia: Chelsea House Publishers, 2006.

2. Fleming A. On the antibacterial action of cultures of a penicillium, with special reference to their use in the isolation of B. influenza. British Journal of Experimental Pathology 1929; 10: 226-236.

3. Sidell S, et al. New antistaphylococcal antibiotics. Comparative in vitro and in vivo activity of cephalothin, nafcillin, cloxacillin, oxacillin, and methicillin. Archives of Internal Medicine 1963; 112: 21-28.

4. Jevons MP. 'Celbenin'-resistant staphylococci. British Medical Journal 1961; 1: 124-125.

5. David MZ, Daum RS. Community-associated methicillin-resistant Staphylococcus aureus: epidemiology and clinical consequences of an emerging epidemic. Clinical Microbiology Reviews 2010; 23: 616-687.

6. Herold BC, et al. Community-acquired methicillinresistant Staphylococcus aureus in children with no identified predisposing risk. Journal of the American Medical Association 1998; 279: 593-598.

7. Hersh AL, et al. National trends in ambulatory visits and antibiotic prescribing for skin and soft-tissue infections. Archives of Internal Medicine 2008; 168: 15851591.

8. David MZ, et al. Predominance of methicillin-resistant Staphylococcus aureus among pathogens causing skin and soft tissue infections in a large urban jail: risk factors and recurrence rates. Journal of Clinical Microbiology 2008; 46: 3222-3227.

9. Manfredi R, Calza L, Chiodo F. Epidemiology and microbiology of cellulitis and bacterial soft tissue infection during HIV disease: a 10-year survey. Journal of Cutaneous Pathology 2002; 29: 168-172.

10. Imaz A, et al. Community associated methicillin-resistant Staphylococcus aureus in HIV-infected patients. AIDS Reviews 2010; 12: 153-163.

11. Popovich KJ, et al. Community-associated methicillinresistant Staphylococcus aureus and HIV: intersecting epidemics. Clinical Infectious Diseases 2010; 50: 979987.

12. Graber CJ, et al. Recurrence of skin and soft tissue infection caused by methicillin-resistant Staphylococcus aureus in a HIV primary care clinic. Journal of Acquired Immune Deficiency Syndromes 2008; 49: 231-233.

13. Crum-Cianflone N, Weekes J, Bavaro M. Recurrent community-associated methicillin-resistant Staphylococcus aureus infections among HIV-infected persons: incidence and risk factors. AIDS Patient Care and STDs 2009; 23: 499-502.

14. Shadyab AH, Crum-Cianflone NF. Methicillin-resistant Staphylococcus aureus (MRSA) infections among HIV-infected persons in the era of highly active antiretroviral therapy: a review of the literature. HIV Medicine 2012; 13: 319-332.

15. Cole J, Popovich K. Impact of community-associated methicillin resistant Staphylococcus aureus on HIVinfected patients. Current HIVIAIDS Reports 2013; 10: 244-253.

16. Vyas KJ, et al. Trends and factors associated with initial and recurrent methicillin-resistant Staphylococcus aureus (MRSA) skin and soft-tissue infections among HIV-infected persons: an 18-year study. Journal of the International Association of Providers of AIDS Care 2013.

17. Lee NE, et al. Risk factors for community-associated methicillin-resistant Staphylococcus aureus skin infections among HIV-positive men who have sex with men. Clinical Infectious Diseases 2005; 40: 1529 1534.

18. Hemmige $\mathbf{V}$, et al. Predictors of skin and soft tissue infections in HIV-infected outpatients in the community-associated methicillin-resistant Staphylococcus aureus era. European Journal of Clinical Microbiology and Infectious Diseases 2015; 34: 339-347. 
19. Mathews WC, et al. Incidence of and risk factors for clinically significant methicillin-resistant Staphylococcus aureus infection in a cohort of HIV-infected adults. Journal of Acquired Immune Deficiency Syndromes 2005; 40: 155-160.

20. Crum-Cianflone NF, Burgi AA, Hale BR. Increasing rates of community-acquired methicillin-resistant Staphylococcus aureus infections among HIV-infected persons. International Journal of STD \& AIDS 2007; 18: $521-526$.

21. Anon. Guidelines for the prevention and treatment of opportunistic infections in HIV-infected adults and adolescents: recommendations from the Centers for Disease Control and Prevention, the National Institutes of Health, and the HIV Medicine Association of the Infectious Diseases Society of America.

22. Richter SS, et al. Activity of ceftaroline and epidemiologic trends in Staphylococcus aureus isolates collected from 43 medical centers in the United States in 2009. Antimicrobial Agents and Chemotherapy 2011; 55: 41544160.

23. Hidron AI, et al. Methicillin-resistant Staphylococcus aureus in HIV-infected patients. Infection and Drug Resistance 2010; 3: 73-86.

24. Greenland S, Pearl J, Robins JM. Causal diagrams for epidemiologic research. Epidemiology 1999; 10: 37-48.

25. Suttorp MM, et al. Graphical presentation of confounding in directed acyclic graphs. Nephrology Dialysis Transplantation 2014; 30: 1418-1423.

26. Textor J, Hardt J, Knuppel S. DAGitty: a graphical tool for analyzing causal diagrams. Epidemiology 2011; 22: 745.

27. Greenland S, Robins JM. Identifiability, exchangeability, and epidemiological confounding. International Journal of Epidemiology 1986; 15: 413-419.

28. Richiardi L, Bellocco R, Zugna D. Mediation analysis in epidemiology: methods, interpretation and bias. International Journal of Epidemiology 2013; 42: 15111519.
29. Mocroft A, et al. Is it safe to discontinue primary Pneumocystis jiroveci pneumonia prophylaxis in patients with virologically suppressed HIV infection and a CD4 cell count $<200$ cells/ $\mu 1$ ? Clinical Infectious Diseases 2010; 51: 611-619.

30. Cox DR. Regression models and life tables. Journal of the Royal Statistical Society: Series B 1972; 34: 187-220.

31. Ai C, Norton EC. Interaction terms in logit and probit models. Economics Letters 2003; 80: 123-129.

32. Group TISS. Initiation of antiretroviral therapy in early asymptomatic HIV infection. New England Journal of Medicine 2015; 373: 795-807.

33. Eldred LJ, et al. Adherence to antiretroviral and pneumocystis prophylaxis in HIV disease. Journal of Acquired Immune Deficiency Syndromes 1998; 18: 117-125.

34. Lepage B, et al. Estimating controlled direct effects in the presence of intermediate confounding of the mediator-outcome relationship: comparison of five different methods. Statistical Methods in Medical Research. Published online: 14 October 2014. doi:10.1177/ 0962280212461194.

35. Williamson EJ, et al. Introduction to causal diagrams for confounder selection. Respirology 2014; 19: 303-311.

36. Robins JM, et al. G-estimation of the effect of prophylaxis therapy for Pneumocystis carinii pneumonia on the survival of AIDS patients. Epidemiology 1992; 3: 319-336.

37. Robins JM, Hernan MA, Brumback B. Marginal structural models and causal inference in epidemiology. Epidemiology 2000; 11: 550-560.

38. Kelly PJ, Lim LL. Survival analysis for recurrent event data: an application to childhood infectious diseases. Statistics in Medicine 2000; 19: 13-33.

39. Popovich KJ, et al. Community-associated methicillinresistant Staphylococcus aureus colonization burden in HIV-infected patients. Clinical Infectious Diseases 2013; 56: 1067-1074. 